\title{
Atomic Force Microscopy for the Study of Diseases: Minireview
}

\author{
Jose Jorge Ortez Toro and Fernando Calvo Gracia* \\ Department of Endocrinology, Spain
}

*Corresponding author: Fernando Calvo Gracia, Endocrinology Department, Hospital Clínico Universitario Lozano Blesa, Zaragoza, Spain

\section{ARTICLE INFO}

Received: 幽 November 08, 2019

Published: 幽 November 18, 2019

Citation: Jose Jorge Ortez Toro, Fernando Calvo Gracia. Atomic Force Microscopy for the Study of Diseases: Minireview. Biomed J Sci \& Tech Res 22(5)-2019. BJSTR. MS.ID.003827.

Abbreviations: AFM: Atomic Force Microscopy; FDA: Food and Drug Administration; EMA: European Medicines Agency

\begin{abstract}
Atomic Force Microscopy (AFM) is an innovative technique very useful for medicine due to its ability to combine it with other cut-of-edge techniques or tools and also because is the only nanoscopic technique capable of studying biological samples in the air or in aqueous medium with nanometric resolution, which opened us a range of possibilities in characterization and application, allowing us to perform things that we previously thought impossible. Noteworthy that the evolution of deadly diseases is exponential, from multi-drug-resistant infectious microorganisms to the incurable cancers, being here where the AFM becomes one of the most important tools finding solutions to these problems, deciphering the mechanisms and molecules involved improving diagnosis and treatment in these pathologies. Here we review the principles of AFM and the fascinating medical opportunities offered by the rapid advances in AFM.
\end{abstract}

Keywords: Nanotechnology; Nanomedicine; Nanoscience; Atomic Force Microscopy; Diseases

\section{Introduction}

AFM is a three dimensional high resolution topographic technique with the ability to observe, manipulate and explore the components of biological cell at sub nanometric resolution, becoming a multifunctional toolbox for the study of live organism, giving us the opportunity to study, discover and understand a lot of functions, molecular interactions and characteristics of live organisms [1,2]. Tissue and cells morphology in disease research is very important because evidence suggests there is a strong link between the mechanical properties of cells and tissues with pathogenic states. Thus, methods that perform concomitant measurements of chemical/biochemical characteristics with physical properties, present considerable opportunities in biomedical research [1]. Molecular interactions make up the basic language of all biological processes, also control the way that cells communicate with each other and form higher-ordered organisms. Remarkably, AFM can measure interactions between and within single biomolecules, giving us clues about the cell adhesion process.

Such information is important for understanding tissue development, tumor metastasis, bacterial infection and to find answers to a lot of medical and biotechnological questions [2]. Medicine has been a very important area of common interest for investigators because of the complexity of the human body and for many years we have been trying to understand how our body works at molecular level, but we had reached our limit; discoveries and developments in medicine had slowed down in the last century and were not evolving at the same speed as the diseases, we were losing the battle against illness, so we had to look for new approaches, new methods, new options to fight back and AFM brought us opportunities to unravel and understand cellular mechanism and interactions, which opens new avenues for medical characterization, diagnosis and treatment.

\section{Atomic Force Microscopy}

Originally invented for topographic imaging, it has evolved into a multifunctional biological toolkit with great versatility, becoming in something much more important than a high resolution microscopy technique because of its ability to reconstruct force maps that can be used to explore nanomechanical properties of microorganism and at the same time, the morphological and 
mechanical modifications induced by external stimuli or different kind of chemical and molecular interactions [3]. AFM relies on the detection of attractive or repulsive surface forces by a sharp tip that is attached to a flexible cantilever1 and can be moved in the $x, y$, and $\mathrm{z}$ direction thanks to a piezoelectric ceramic. The deflection of the cantilever is monitored on a 4 squares photodiode which is an optical system used to reconstruct the topography of the sample [46]. The Basic instrumental setup of an AFM is illustrated in Figure 1. Laser light is reflected from the end of the cantilever onto the photodiode position-sensitive-detector as we can see in Figure 1.

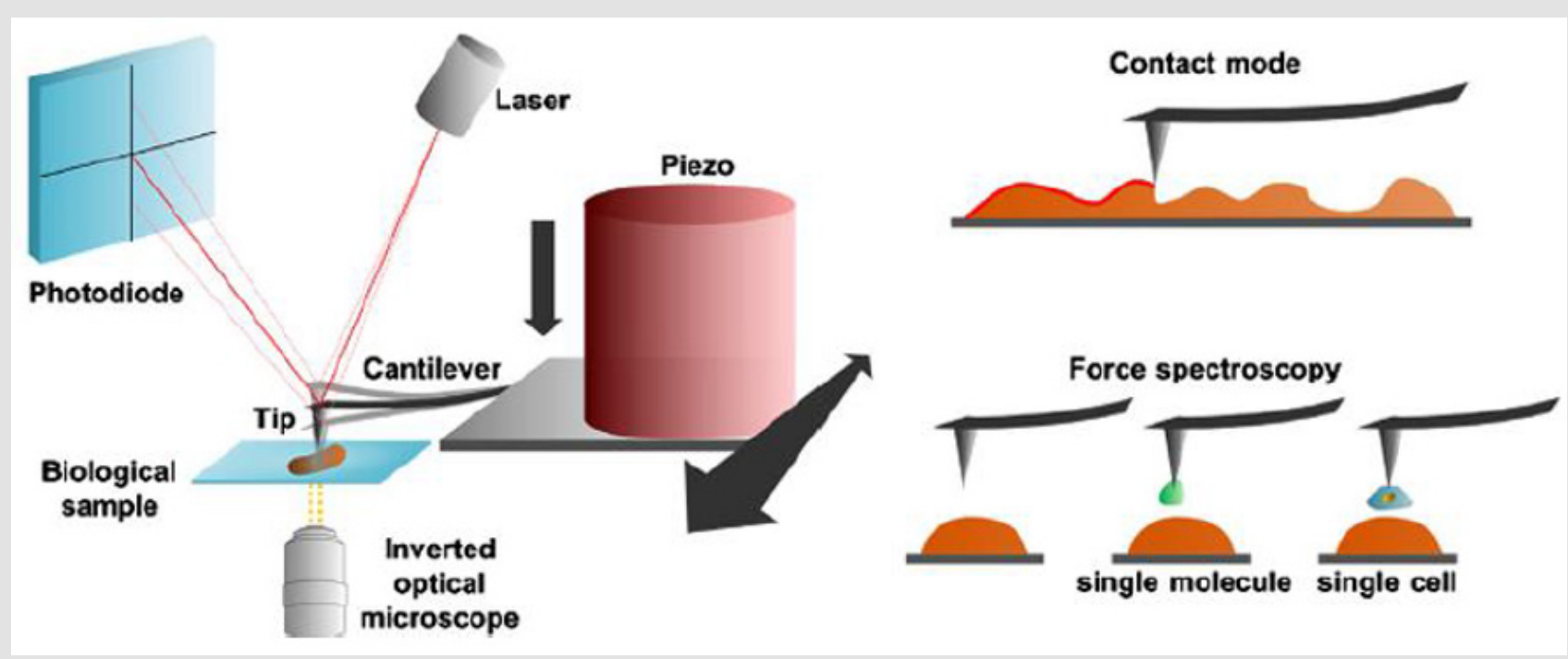

Figure 1: Atomic Force Microscopy technology and some of its operational modes.

The AFM can be used to produce topographical images (like in contact mode) or to measure forces (in the force spectroscopy mode) between a bare or a functionalized tip (with a biomolecule or a single cell) and the sample, like the example in Figure 1 [6]. The AFM tips and cantilever are microfabricated from silica or silicon nitride, which should have contact or near contact with the surface of interest [4]. The diversity of operational modes in AFM offers the ability to directly measure protein-protein interactions, determine locations and distributions of certain binding sites, and measure stiffness among others. There are many different modes for using AFM depending on the type of sample, morphology, physical/ biochemical characteristics that we are interested in studying, we can even combine it with other cut-of-edge techniques like Near-field scanning optical microscopy, Fluorescence microscopy, Scanning electrochemical microscopy among others, becoming in novel hybrid AFM techniques which opens up a range of possibilities in characterization and application in different fields, especially in medicine

\section{AFM Studying Diseases}

AFM has emerged as a robust well-tested method to image and probe living systems [1]. It has, improving and becoming essential in the study and investigation of diseases [1,3]. We can classify its use in 2 big groups, first, we have characterization, especially used in translational medicine for the investigation of different unknown mechanisms, ligands, receptors, antibodies, proteins, among others, then we have the more interesting area from the clinical perspective, the application group, where we can see many breakthroughs discoveries in diagnosis, treatment and nanodevices development, for example, the use of nanoparticles as carriers or the used of bioengineered nanoparticles to act in specific receptors, organs or cells, the creation of new diagnosis techniques or the construction of novel smart devices for the benefit of human health, all these using AFM with other innovative techniques [1].

\section{Clinical Applications}

The versatility of AFM and its capacity to be easily hybridized with secondary techniques make it one of the best tools for studying diseases in both, characterization and application [1]. Here we will discuss some clinical applications and examples. In the virus research field, the main drawback is its size because they are one of the smallest infecting organisms known to science this added to its versatility and constant evolution becomes a timebomb that already has killed millions of people in the past and can do so in the future. That is why the AFM has brought light to this dark and troubling area, allowing us to learn and discover more about these infectious agents throughout many ongoing studies obtaining images, pathogenic mechanisms, ligands, physical/ biochemistry properties of different viruses like smallpox, Herpes simplex, influenza, chikungunya, among others, stimulating future drugs development or novel diagnosis tools [1]. There are also a lot of applications with AFM to the bacterial field.

In one hand it has been studied the morphology and behavior of bacteria alone or interacting with their environment and in another hand, we have the pharmacological studies that have been performed on different multiresistant bacteria, with novel antibiotics and antimicrobial peptides, engineered nanoparticles using known 
antibiotics but becoming in an entirely different molecule with completely new characteristics and action spectrum contributing to save millions of lives and giving a whole range of innovative possibilities [6]. Furthermore, an area of remarkable growth is the creation of bioengineer new materials, nano-scaffolds, nanoparticles designs (carbon nanotubes, gold nanoparticles, etc), used in a lot of novel devices for example for coating orthopedics external fixators or to prevent wound infections, to coat central ways with integrated anticoagulants, among others things, many of them already been used in clinical practice, other in developmental phase, but still, with excellent results. Noteworthy that many breakthrough discoveries have been made in the last decade in cancer diagnosis and cancer treatment using AFM, for example in prostate cancer it was revealed new interactions and properties of the cancer cells, giving some insight on how they metastasize, helping to identify new therapeutic targets for future treatment options.

In bladder cancer, it was found new information about the forces involved in the interactions between bladder cancer cells and the endothelium, concluding that cancer cells and normal cells exhibit remarkable and measurable differences seeing only by AFM spectroscopy which is going to lead to new diagnosis and therapeutic targets. In fact, in these two areas (cancer and infections) we have the major concentration of FDA/EMA approved drugs that were developed through nanotechnology and also the new investigated applications under clinical trials for future approval. Moreover, in the devastating neurodegenerative diseases world, there are many conducting studies with AFM to understand more about them, providing new therapeutic targets and understanding the mechanisms that produce it, so that one day we can prevent them and treat them properly $[7,8]$. In addition, in the areas of rheumatology and orthopedics it has been possible to detect early changes in the structure and mechanical properties of normal ageing and osteoarthritic articular cartilage in mice and patients, using AFM at the nanometer scale [9].

Consequently, has opened up the exciting prospect of using a simple nanodevice based on AFM as a potential clinical tool, allowing the direct arthroscopic inspection of joints [9]. Further, inhaled formulations are a popular way of treating the symptoms of respiratory diseases because the drug is delivered directly to the site of action and AFM is essential to identify the characteristics of dry powder formulations, like surface morphology, surface energetics, rugosity and for determining the interparticle forces between the drug and the carrier nanoparticles, proven to be extremely valuable in developing new drugs [10]. AFM is evolving and is going to be an area of constant growth, enabling breakthroughs in science and medicine in many medical specialities, with noteworthy researches in dermatology, endocrinology, pharmacology, neurology, among a lot of other areas of interest. Last but not least, its well known that nanoparticles drug delivery systems have been used in the clinic since the early 1990's, but in the last 30 years the field of nanomedicine has evolved dramatically. Currently, there are 28 FDA/EMA approved nanomedicines and 115 ongoing clinical trials evaluating different nanoparticles for medical applications and from these, 75 clinical trials began in the last 3 years, we highlight this to provide a brief summary of the current situation [11].

\section{Conclusion and Perspectives}

AFM is one of the most revolutionary and important techniques in the world of science and especially in biology, allowing us to obtain images of a sample or specimen alive or dead, in aqueous medium, air or natural environment at nanometric scale, opening a gap of possibilities and opportunities for study and understanding the "nanoscopic" world [6]. The new approaches that AFM brings us in medicine are exciting because is a tool that enables us to understand many interactions or diseases that in medicine we called "idiopathic" (of unknown cause), also there are a lot of medical treatments of unknown mechanism. The exponential development, mutation and evolution of infectious microorganisms is winning the battle against us, every day we have more multidrug-resistant bacteria, mutated viruses and deadly degenerative diseases that takes millions of lives worldwide and AFM have proven to be an effective tool for the discovery of new diagnostic and therapeutic targets. The main challenges that nanomedicine is facing today include uncertainties concerning safety, regulatory and ethical requirements in the field, but is also changing and evolving presenting new data, laws, guidelines and new regulations bringing some light into this trending topic [12].

In the near future, we have to stimulate the development of multidisciplinary techniques like AFM, until it will become a daily tool in all sciences because this will provide more ideas and investigations that will lead to more discoveries and breakthroughs. Today we have a whole new world of possibilities in front of us, especially for researchers and we need to encourage the study and update in this area, because one of the main issues in this topic is the lack of multidisciplinary teams that include medical doctors, translational investigators, engineers, chemists, physicists, biologists, among many other scientists, who contribute and unify their knowledge for the common good and evolution of science heading toward a personalized design and engineer medicine.

\section{References}

1. Morton KC, Baker LA (2014) Atomic force microscopy-based bioanalysis for the study of disease. Anal Methods 6: 4932-4955.

2. Müller DJ,DufrêneYF (2008) Atomicforcemicroscopy as a multifunctional molecular toolbox in nanobiotechnology. Nat Nanotechnol 3.

3. Longo G, Kasas S (2014) Effects of antibacterial agents and drugs monitored by atomic force microscopy. WIREs Nanomedicine and Nanobiotechnology 6: 230-244

4. Vahabi S (2013) Atomic Force Microscopy Application in Biological Research: A Review Study. IJMS 38(2): 76-83.

5. Kasas S, Thomson NH, Smith BL, Hansma PK, Miklossy J, et al. (1996) Biological Applications of the AFM : From Single Molecules to Organs. Intenational Journal of Imaging Systems 8: 151-361. 
6. Pillet F, Chopinet L, Formosa C, Dague É (2014) Atomic Force Microscopy and pharmacology: From microbiology to cancerology. Biochimica et Biophysica Acta 1840(3): 1028-1050.

7. Drolle E, Hane F, Lee B, Leonenko Z (2014) Atomic force microscopy to study molecular mechanisms of amyloid fibril formation and toxicity in Alzheimer's disease. Drug Metab Rev 46(2): 207-223.

8. Juan Ren, Huarong Huang, Yue Liu, Xi Zheng, Qingze Zou, et al. (2015) An Atomic Force Microscope Study Revealed Two Mechanisms in the Effect of Anticancer Drugs on Rate-Dependent Young's Modulus of Human Prostate Cancer. PLoS One.

\section{ISSN: 2574-1241}

DOI: 10.26717/BJSTR.2019.22.003827

Fernando Calvo Gracia. Biomed J Sci \& Tech Res

(C) This work is licensed under Creative Commons Attribution 4.0 License

Submission Link: https://biomedres.us/submit-manuscript.php
9. Stolz M, Gottardi R, Raiteri R, Miot S, Martin I, et al. (2009) Early detection of aging cartilage and osteoarthritis in mice and patient samples using atomic force microscopy. Nat Nanotechnol 4(3): 186-192.

10. Weiss C, Mcloughlin P, Cathcart H (2015) Characterisation of dry powder inhaler formulations using atomic force microscopy. Int J Pharm 494(1): 393-407.

11. Anselmo AC, Mitragotri S (2019) Nanoparticles in the clinic: An update. Bioeng Transl Med 4(3): 1-16.

12.Zingg R, Fischer M (2019) The consolidation of nanomedicine. Wiley Interdiscip Rev Nanomedicine Nanobiotechnology 11(6): 1-6.

$\begin{array}{ll}\text { BIOMEDICAL } & \text { Assets of Publishing with us } \\ \text { RESEARCHES } & \text { - Global archiving of articles } \\ \text { - Immediate, unrestricted online access } & \text { - Rigorous Peer Review Process } \\ & \text { - Authors Retain Copyrights } \\ \end{array}$

